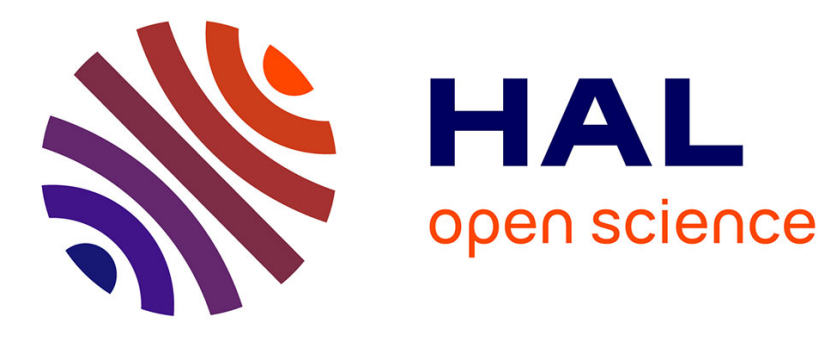

\title{
Vibrational coherence and quantum yield of retinal-chromophore-inspired molecular switches
}

Moussa Gueye, Marco Paolino, Etienne Gindensperger, Stefan Haacke, Massimo Olivucci, Jérémie Léonard

\section{- To cite this version:}

Moussa Gueye, Marco Paolino, Etienne Gindensperger, Stefan Haacke, Massimo Olivucci, et al.. Vibrational coherence and quantum yield of retinal-chromophore-inspired molecular switches. Faraday Discussions, 2020, 10.1039/c9fd00062c . hal-02394778

\section{HAL Id: hal-02394778 \\ https://hal.science/hal-02394778}

Submitted on 29 Nov 2020

HAL is a multi-disciplinary open access archive for the deposit and dissemination of scientific research documents, whether they are published or not. The documents may come from teaching and research institutions in France or abroad, or from public or private research centers.
L'archive ouverte pluridisciplinaire HAL, est destinée au dépôt et à la diffusion de documents scientifiques de niveau recherche, publiés ou non, émanant des établissements d'enseignement et de recherche français ou étrangers, des laboratoires publics ou privés. 


\title{
Vibrational coherence and quantum yield of retinal-chromophore-inspired molecular switches
}

\author{
Moussa Gueye, ${ }^{1}$ Marco Paolino, ${ }^{2}$ Etienne Gindensperger, ${ }^{3}$ \\ Stefan Haacke, ${ }^{1}$ Massimo Olivucci, ${ }^{2,4,}{ }^{*}$ Jérémie Léonard. ${ }^{1, *}$ \\ ${ }^{1}$ Université de Strasbourg, CNRS, Institut de Physique et Chimie des Matériaux de Strasbourg, UMR \\ 7504, F-67034 Strasbourg, France. \\ ${ }^{2}$ Dipartimento di Biotechnologie, Chimica e Farmacia, Università di Siena, I-53100 Siena, Italy. \\ ${ }^{3}$ Université de Strasbourg, CNRS, Laboratoire de Chimie Quantique, Institut de Chimie, UMR 7177, F- \\ 67008 Strasbourg, France \\ ${ }^{4}$ Department of Chemistry, Bowling Green State University, Bowling Green, $\mathrm{OH} 43403$, USA. \\ *molivuc@bgsu.edu, Jeremie.Leonard@ipcms.unistra.fr
}

\begin{abstract}
UV-Vis transient absorption (TA) spectroscopy is used to carry out a systematic investigation of the ultrafast $\mathrm{C}=\mathrm{C}$ double photoisomerization dynamics and quantum yield of each isomer of a set of six chromophores based on the same retinal-inspired, indanylidene pyrrolinium (IP) molecular framework. All compounds undergo a sub-picosecond photoisomerization, and can be categorized within two photoisomerization scenarios. Scenario I corresponds to compounds which display the signatures of a vibrationally coherent reactive motion through the conical intersection, with different degrees of vibrational coherence. Qualitatively distinct TA signatures are observed for other compounds which are therefore proposed to obey scenario II referring to an intermediate regime between scenario I and a thermally-equilibrated, fully stochastic photoreaction. Remarkably, the photoisomerization scenario is observed to correlate with the computed distortion from planarity of the ground state equilibrium geometry reflecting the torsional strain that would be released after photoexcitation. The most planar compounds - i.e. having a $\mathrm{C}=\mathrm{C}$ double bond pre-twist of less than $10^{\circ}$ - obey scenario II, while compounds obeying scenario I have larger pre-twists. The most pre-twisted compounds $\left(>15^{\circ}\right)$ show pronounced oscillatory signatures of a reaction-induced, low-frequency vibrational wavepacket observed in the $S_{0}$ photoproduct and assigned to the torsion mode of the reaction coordinate, thus mimicking the vibrationally coherent photoisomerization dynamics of the rhodopsin protein. Importantly, the systematic comparison of all photoisomerization quantum yields does however not reveal any correlation with observables such as excited state life time, vibrational coherence, absorption wavelengths or degree of pre-twisting.
\end{abstract}

\section{Introduction}

Double-bond photoisomerization has long been exploited in man-tailored light-driven molecular switches and single-molecule motors for providing the opto-mechanical energy conversion necessary to implement their functions. [1-3] In such molecular devices, a high photoisomerization quantum yield ( $Q Y$ ) is desirable to enhance the function efficiency - e. g. in terms of conversion of photon energy in mechanical motion at the population level. So far, however, there is no chemical design criterion for controlling and maximizing the photoisomerization QY of synthetic molecules. In contrast, natural evolution, has been able to develop photo-responsive proteins, in which the photoisomerization of a $\mathrm{C}=\mathrm{C}$ double bond triggers a biological function with high quantum efficacy. A paradigmatic example is that of bovine Rhodopsin (Rho), the vertebrate photodetector for dim-light vision, in which the 11-cis 
protonated Schiff base of its retinal (PSBR) chromophore undergoes a particularly fast ( $<200 \mathrm{fs})$, [4] unidirectional and stereospecific photoisomerization around the $\mathrm{C} 11=\mathrm{C} 12$ double bond producing the all-trans isomer [5] with a 67\% QY. [6] Noticeably, when 11-cis PSBR is free in solution the same alltrans photoproduct is formed, but with a $20 \%$ QY $[7,8]$ and a much longer excited state life time (3 to $4 \mathrm{ps)}$ irrespective of the specific solvent environment. $[9,10]$ Hence the interaction between the PSBR chromophore and the cavity of the Rho protein appears to control the reactivity and enhance the QY of PSBR photoisomerization. A major effect of this interaction is to turn the PSBR isomerization into a vibrationally coherent photoisomerization, [11-13] meaning that the energy of the absorbed photon is efficiently funneled into the isomerization coordinate on a time scale faster than vibrational dephasing. This unique property is argued to be a prerequisite for optimizing the photoisomerization quantum yield of Rho. [14] Indeed, the PSBR isomerization is mediated by decay through a conical intersection (CInt) which corresponds to a nearly $90^{\circ}$ twisted conformation of the $\mathrm{C}=\mathrm{C}$ isomerizing bond, where excited $\left(S_{1}\right)$ and ground $\left(S_{0}\right)$ electronic states become degenerate. It has been predicted $[15,16]$ and recently supported experimentally, [14] that the phase of the oscillatory motion of a specific vibrational mode - namely the hydrogen-out-of-plane $\left(\mathrm{HOOP}, \sim 900 \mathrm{~cm}^{-1}\right)$ mode of the reactive $\mathrm{HC}=\mathrm{CH}$ moiety of the chromophore - controls the branching at the Clnt (more precisely, at the entire set of accessible CInt's belonging to the intersection space) between (i) completion of the $\mathrm{C}=\mathrm{C}$ bond twisting up to $180^{\circ}$ and formation of the all-trans isomer or (ii) abortion of the reactive process leading to the reconstitution of the initial 11-cis isomer.

In order to mimic the outstanding photoreaction properties of PSBR in Rho, a molecular photoswitch based on the indanylidene-pyrroline (IP) skeleton was synthesized $[17,18]$ such that, in solution, the $\pi$-electron system, molecular framework and conjugating substituents of IP would mimic the topographies of the potential energy surfaces and CInt of 11-cis PSBR in Rho. Indeed, the design strategy was guided by the computational modeling of the electronic structure and photoisomerization dynamics of the IP's in solution, using QM/MM models based on a multi-configurational wavefunction describing the excitation of the ten $\pi$-electrons of the IP backbone at the CASSCF and CASPT2 quantum chemistry levels (QM, Quantum Mechanics) and the solvent at the Molecular Mechanics (MM) level. [18-23] The photoisomerization of the so-called "parent" methoxy-N-alkylated IP (MeO-NAIP) was shown to occur on a 300-fs time scale.[19] Remarkably, the spectroscopic signatures of a Rhodopsinlike vibrationally coherent motion were observed in several representatives of the MeO-NAIP family in solution. These are the first synthetic molecular switches displaying a vibrationally coherent photoisomerization. $[21,24,11,25]$ Recently, we demonstrated [23] that a pre-twist of the isomerizing $\mathrm{C}=\mathrm{C}$ bond in the ground state $\left(\mathrm{S}_{0}\right)$ is enough to engineer a vibrationally coherent photoisomerization in MeO-NAIP and, therefore to achieve a truly biomimetic design strategy. This is an important result, because it reveals a mechanism which could have been adopted by Rho to engineer the vibrationally coherent photoisomerization of the PSBR chromophore. However, while in Rho this coherence property has been connected to the optimization of its QY, [14] the photoisomerization QY of MeONAIP remains no larger than $21 \%,[18,19,26]$. It is therefore evident that, mechanistic factors beyond the vibrational coherence alone, are involved in the control of the QY.

In the context of molecular-level opto-mechanical energy transduction, the IP compounds are a good starting point for the fundamental investigation of ultrafast $\mathrm{C}=\mathrm{C}$ photoisomerization towards designing innovative molecular switches and motors, [27] while targeting the mechanistic factors leading to optimal QY's, possibly exploiting the demonstrated vibrational coherence. [28] So far, functional 
applications of the IP or other retinal-inspired compounds have been explored towards dipole moment photoswitching [20] or peptide structure photocontrol. $[29,30]$ In addition, in chiral compounds or chiral environments the mirror image symmetry is broken which is expected to result in a unidirectional rotary motion, the fundamental property of a single-molecule rotary motor. This effect has been investigated computationally and predicted in PSBR analogues. [31-33] Consequently, chiral NAIP compounds have been synthesized to explore their applications as light-driven molecular motors. [34]

In this paper, we review and expand our UV-Vis transient absorption (TA) spectroscopy investigations of the photoreactivity of the set of IP compounds displayed in Figure 1A, with a special focus on photoisomerization QYs. The methodology used for determining and comparing the absolute QYs of all compounds is reported in Section II. As a very general rule driving their early photophysics, the $S_{1}$ state of the $-\mathrm{C}=\mathrm{NR}(+)$ - terminating polyenes $(\mathrm{R}=\mathrm{H}$, alkyl) like IP or PSBR features a charge-transfer character. [35] In IP, the positive charge located on the pyrolinium moiety in $\mathrm{S}_{0}$ translocates towards the indanylidene moiety when the system is promoted to $S_{1}$. Therefore, as previously discussed [18], the electron-rich $\mathrm{MeO}$ group on $\mathrm{C6}^{\prime}$ ' of the indanylidene moiety stabilizes the IP $\mathrm{S}_{1}$ state. Here we report on the NAIP compound (see Figure $1 \mathrm{~A}$ ), where this $\mathrm{MeO}$ group has been removed. Although its $\mathrm{S}_{0}$ absorption spectrum is blue shifted (see Figure 1B), indicating a significant destabilization of the $S_{1}$ charge distribution as expected, its photoisomerization dynamics is similar and even faster than that of the parent MeO-NAIP, including marked signatures of vibrational coherence along the reaction coordinate. Therefore, after an experimental Section II, we describe in Section III the signatures of the vibrationally coherent reaction scenario - hereafter named scenario I - already observed in MeO-NAIP $[21,23]$ and its zwitterionic version ZW-MeO-NAIP (see Figure $1 A$ ), [24] by illustrating them with the TA data recorded on the novel NAIP compound. 
(A)<smiles>COc1ccc2c(c1)CC(C)(C)C2=C1CCN(C)[C@@H]1C</smiles>

Z-NAIP

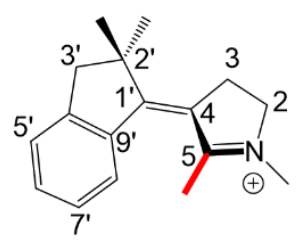

Z-ZW-MeO-NAIP<smiles>COc1ccc2c(c1)CC(C)(C)C2=C1CC(C(=O)[O-])N(C)[C@@H]1C</smiles>

E-dMe-MeO-NAIP<smiles>COc1ccc2c(c1)CC(C)(C)C2=C1CCN(C)C1</smiles>

E-Ch-MeO-NAIP<smiles></smiles>

E-Ch-dMe-MeO-NAIP<smiles>COc1ccc2c(c1)CC(C)(C)[Te]2=C1CCN(C)C1</smiles>

(B)

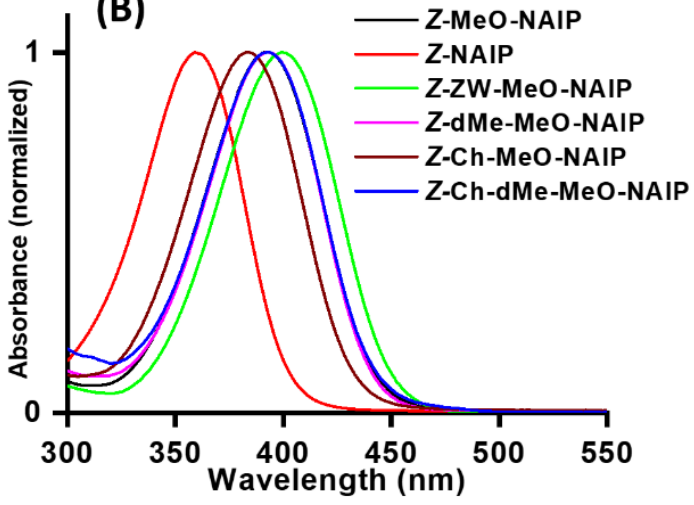

(C)

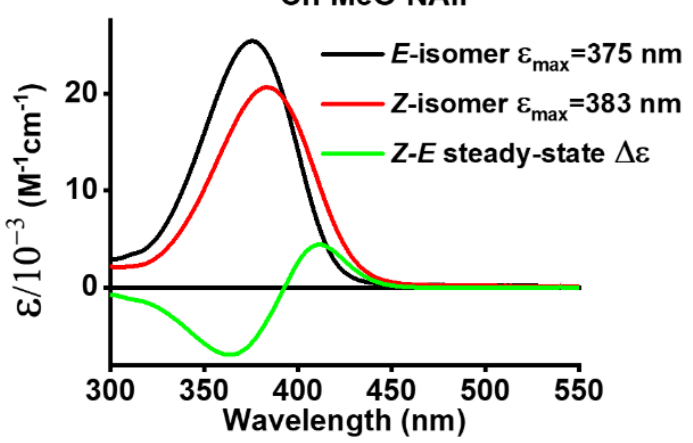

Figure 1 (A) Chemical structure of the six investigated molecular photoswitches in their most stable ground state isomer and (B) the normalized steady state absorption spectra of their $Z$ isomers in methanol. (C) Molar extinction $(\varepsilon)$ spectra of the pure $E$ (black) and $Z$ (red) isomers of Ch-MeO-NAIP in $\mathrm{MeOH}$ together with their difference $\Delta \varepsilon$ (green).

Besides the $S_{1}$ charge-transfer character, another central ingredient of the biomimetic design is the intramolecular steric hindrance imposed by the two methyl groups on C2' and the methyl group on C5. Altogether they impose the $Z$ configuration of the parent MeO-NAIP to be the most stable $\mathrm{S}_{0}$ isomer, as well as a non-planar geometry. Upon removing the methyl group on $\mathrm{C} 5$, we produce the "demethylated" compound (dMe-MeO-NAIP). [36] Due to the reduced steric hindrance, its most stable $\mathrm{S}_{0}$ isomer becomes the $E$-isomer which acquires a substantially planar geometry. In dMe-MeO-NAIP, the characteristic TA signatures of a vibrationally coherent photoreactive motion are no longer observed, pointing to a central role of the $\mathrm{C}=\mathrm{C}$ double bond pre-twist in engineering a vibrationally coherent photoisomerization. [23] The photoisomerization scenario of dMe-MeO-NAIP is therefore qualitatively distinct from the vibrationally coherent scenario I introduced above, and will therefore be named scenario II hereafter. Another way of releasing the intramolecular steric hindrance is to replace one methyl group on C2' by a hydrogen atom, which results in a chiral compound since C2' becomes a stereogenic center. Such a chiral version of the above demethylated compound, i.e. ChdMe-MeO-NAIP, has been reported separately [34] and behaves like its achiral version, i.e. according to photoisomerization scenario II. Here, we report for the first time on the chiral version of the parent, C5-methylated, MeO-NAIP compound, i.e Ch-MeO-NAIP. We observe, that the $Z$ (less planar) isomer of Ch-MeO-NAIP behaves according to scenario I, but its $E$ (more planar) isomer to scenario II. This is so far the only IP compound which displays an isomer-specific photoisomerization scenario. In Section 
IV, we will illustrate the difference between photoisomerization scenarios I and II with the TA data recorded on both isomers of Ch-MeO-NAIP.

In Section V, we compile and summarize the photoisomerization properties of all IP compounds investigated so far in this and previous works. Furthermore, the modelling of the $\mathrm{S}_{0}$ equilibrium structure of the entire set of compounds supports the existence of a correlation between the magnitude of the deviation from planarity - due to changes in the substitution pattern - and the observation of the spectroscopic signatures of a vibrationally coherent photoisomerization motion (scenario I or II). In contrast, we observe that while all measured QY's range from $13 \%$ to $35 \%$, no correlation can be detected with observed or computed quantities such as excited state life time, vibrational coherence, absorption wavelengths or degree of pre-twisting.

\section{Material and Methods}

The synthesis of all IP compounds displayed in Figure 1A is described in detail in the Supplementary Information (SI). To ensure comparability, all spectroscopic investigations reported here were carried out at room temperature on compounds dissolved in methanol $\left(\mathrm{CH}_{3} \mathrm{OH}\right)$. The $Z$ :E isomer content of each IP solution is given in Table 1 , as determined by ${ }^{1} \mathrm{H}$-NMR spectroscopy in deuterated solvent $\left(\mathrm{CD}_{3} \mathrm{OD}\right)$. In the dark at room temperature, the most stable isomer is $Z$ for NAIP, MeO-NAIP and ZWMeO NAIP, but $E$ for dMe-MeO-NAIP, Ch-MeO-NAIP and Ch-dMe-MeO-NAIP. For the first three compounds the $E$ isomer accumulates upon illumination in the red wing of the $\mathrm{S}_{0}-\mathrm{S}_{1}$ absorption band. For the last three compounds, the $Z$ isomer accumulates upon illumination in the blue side of the same band. At room temperature (in the dark), thermal isomerization back to the most stable isomer occurs only for MeO-NAIP and ZW-MeO-NAIP and takes several hours (i.e. it typically occurs overnight). This allows us to perform ${ }^{1} \mathrm{H}-\mathrm{NMR}$ spectroscopy and TA experiments on stationary isomer mixtures and to infer the spectroscopic signatures of the least stable isomer.

Table 1: Z:E Isomer composition of the so-called dark state (DS) and photostationary state (PSS) of all IP solutions investigated in this work, as determined by ${ }^{1} \mathrm{H}$-NMR spectroscopy in deuterated methanol.

\begin{tabular}{|c|c|c|c|}
\hline \multirow{2}{*}{ Compound } & \multicolumn{2}{|c|}{$Z: E$ composition (percentages) } & Reference \\
\cline { 2 - 4 } & Dark state (DS) & $\begin{array}{c}\text { Photostationary state (PSS) } \\
\text { (wavelength) }\end{array}$ & \\
\hline NAIP & $90: 10$ & $37: 63(400 \mathrm{~nm})$ & {$[37]$} \\
\hline MeO-NAIP & $96: 4$ & $12: 88(455 \mathrm{~nm})$ & {$[37]$} \\
\hline ZW-MeO-NAIP & $98: 2$ & $12: 88(455 \mathrm{~nm})$ & {$[37]$} \\
\hline Ch-MeO-NAIP & $1: 99$ & $70: 30(343 \mathrm{~nm})$ & This work \\
\hline dMe-MeO-NAIP & $5: 95$ & $60: 40(343 \mathrm{~nm})$ & This work \\
\hline Ch-dMe-MeO-NAIP & $5: 95$ & $62: 38(343 \mathrm{~nm})$ & This work \\
\hline
\end{tabular}

All compounds have been studied by transient absorption (TA) spectroscopy using a $400 \mathrm{~nm}$ pump / UV-IVs supercontinuum probe set-up with 80-fs time resolution as described in ref [24]. In short, we use a $400 \mathrm{~nm}$ pump pulse obtained by second harmonic generation from an $800 \mathrm{~nm}, 40 \mathrm{fs}$ pulse delivered by a $5 \mathrm{kHz}$ Ti:Sa amplified laser system. In addition, the NAIP compound was also studied using a shorter $400 \mathrm{~nm}$ pulse generated by sum frequency generation of a sub-7 fs red-near IR pulse 
produced by a hollow fiber compressor, $[38,39]$ and resulting here in a sub-30 fs time resolution (data displayed in Figures 2, 3 and 4). In all experiments, a supercontinuum light pulse is generated in $\mathrm{CaF}_{2}$ with the $800 \mathrm{~nm}$ fundamental pulse and split in two beams: one travels through the sample and is detected with a spectrometer to probe the pump-induced change in the sample absorbance, the other is sent to the spectrometer directly and used as a reference beam to correct for the probe intensity fluctuations. The relative linear polarizations of pump and probe beams are set to magic angle $\left(54.7^{\circ}\right)$. The pump-probe delay is scanned using a motorized delay line. Both pump and probe beams are focused and overlapped in a $0.2-\mathrm{mm}$ thick flow cell containing the sample in solution. A peristaltic pump circulates the sample to refresh it between two excitation laser shots. The sample concentration is systematically adjusted to achieve an absorbance $\mathrm{OD}=0.4$ to 0.6 at the absorption maximum on the $200 \mu \mathrm{m}$ sample thickness (i.e. $c \varepsilon_{\max }=20$ to $30 \mathrm{~cm}^{-1}$ ), corresponding to concentrations $c$ in the range of 1 to $2 \mathrm{mM}$ depending on the compounds. The pump intensity is kept in the linear regime of excitation in order to promote the sample to its first excited state $\left(S_{1}\right)$ with a few-percent probability (see an estimate of the excitation probability - averaged of the excitation volume - in the SI). All TA data presented here are post-processed in order to compensate for the group velocity dispersion (GVD) in the probe beam so as to define accurately the time zero at all wavelengths. Also, the transient signals generated in the solvent and the quartz flow cell are subtracted from the data. (For details about GVD and solvent signal corrections, see e.g. the SI of ref [24]).

For TA spectroscopy, each compound was dissolved in $\mathrm{MeOH}$ and the solution split in two. One sample was illuminated until a photostationary state (PSS) is reached, the other one kept in the dark state (DS). Two TA experiments were then performed successively in identical conditions, one on the DS sample and one on the PSS sample. Each sample is a different mixture of isomers, characterized by a fraction $x$ of $Z$ isomer, and an absorbance: $A(x)=c_{0} l\left(x \varepsilon_{Z}+(1-x) \varepsilon_{E}\right)$, with $c_{0}$ the total solute concentration, $\varepsilon_{Z}$ and $\varepsilon_{E}$ the extinction coefficients of the $Z$ and $E$ isomers respectively at the pump wavelength, and $l$ the sample thickness. The isomer fractions $x_{1}$ and $x_{2}$ of both samples are determined by prior ${ }^{1} \mathrm{H}-\mathrm{NMR}$ spectroscopy experiments performed on the dark and illuminated samples prepared in the same conditions. Performing TA experiments on two known $x_{1}$ and $x_{2}$ isomer mixtures allows us to retrieve the isomer-specific differential absorption according to the following formula (see SI):

with

$$
\begin{gathered}
\Delta A_{\mathrm{Z}}(\lambda, t)=\frac{g(1)}{x_{1}-x_{2}}\left(\frac{1-x_{2}}{g\left(x_{1}\right)} \Delta A_{1}(\lambda, t)-\frac{1-x_{1}}{g\left(x_{2}\right)} \Delta A_{2}(\lambda, t)\right) \\
\Delta A_{\mathrm{E}}(\lambda, t)=\frac{g(0)}{x_{2}-x_{1}}\left(\frac{x_{2}}{g\left(x_{1}\right)} \Delta A_{1}(\lambda, t)-\frac{x_{1}}{g\left(x_{2}\right)} \Delta A_{2}(\lambda, t)\right)
\end{gathered}
$$

where $\Delta A_{1}(\lambda, t)$ and $\Delta A_{2}(\lambda, t)$ are the TA signals recorded with sample 1 and 2 characterized by the same total concentration $c_{0}$ but different $Z$ isomer fractions $x_{1}$ and $x_{2}$, and different absorbances $A\left(x_{1}\right)$ and $A\left(x_{2}\right)$ at the excitation wavelength, respectively. The function $g(x)$ accounts for the fact that the sample absorbance $A(x)$ at the pump wavelength and therefore the excitation probability depend on the isomer fraction $x$ (see details in the $\mathrm{SI}$ ).

To determine the photoisomerization quantum yields of all IP isomers, we exploit the TA data recorded at long-enough time delays (typically $>100$ ps) where the photoreaction and subsequent vibrational 
relaxation and thermalization are completed, such that the $\Delta \mathrm{A}(\mathrm{t})$ signals have become stationary, $\Delta \mathrm{A}(\mathrm{t}>100 \mathrm{ps})=\Delta \mathrm{A}^{\text {stat }}$. We could systematically verify that this final $\Delta \mathrm{A}^{\text {stat }}$ spectrum is proportional to the difference $\Delta \varepsilon$ between the $E$ and $Z$ extinction coefficients (see e.g. Figure $1 C$, green spectrum for the $\Delta \varepsilon$ spectrum of Ch-MeO-NAIP). First, this demonstrates that the only photoreaction that occurs is photoconversion from $E$ to $Z$ or vice versa. Second, the ratio $\Delta \mathrm{A}^{\text {stat }} / \Delta \varepsilon$ is wavelength independent and equal to the product of the excitation probability and the photoisomerization quantum yield (QY) at the pump wavelength (see SI for details). The excitation probability is in general not accurately measureable which precludes the determination of an absolute value for the QY. However, when recording successively the TA dataset of two distinct compounds in the same experimental conditions, the relative excitation probabilities of both samples are given by their relative extinction coefficients (which are accurately measured) at the pump wavelength. In this case, the ratio of the photoisomerization quantum yields of both compounds can be accurately determined (see SI for details). Therefore, we performed several acquisition campaigns to compare the amplitudes of the final TA spectra $\Delta \mathrm{A}^{\text {stat }}$ of several compounds while keeping experimental conditions unchanged. We could thus determine accurately the photosiomerization quantum yields of all compounds relative to one another. We finally calibrated all the values to that of the Z-MeO-NAIP which has been measured to be $21 \%$ in two distinct labs using two independent methods. [18, 19]

The ground state equilibrium structure of the IP compounds in methanol was predicted at the DFT level of theory, using the B3LYP functional [40] with dispersion correction [41] and employing the 6$31 \mathrm{G}^{* *}$ basis set. Solvent contribution was included using a polarizable continuum model (PCM). [42] The Gaussian09 quantum chemistry package [43] was used for these calculations. 


\section{NAIP: illustration of photoisomerization scenario I}

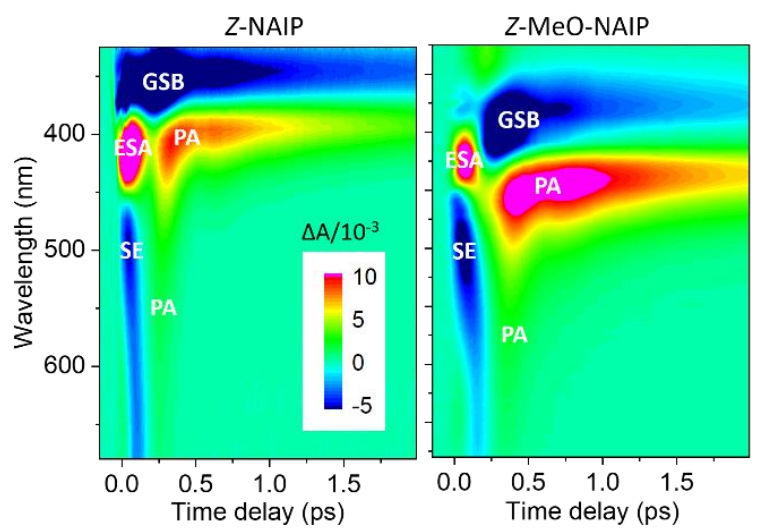

Figure 2: Pump-induced transient absorption 2D maps ( $\Delta A$ in false color code) of the $Z$ isomers of NAIP (left) and MeO-NAIP (right) as a function of wavelength ( $\mathrm{nm}$ ) and time delay (ps) after excitation at $400 \mathrm{~nm}$. Negative $\Delta \mathrm{A}$ signals are coded in blue and assigned to stimulated emission (SE, at early times and $\lambda>450 \mathrm{~nm}$ ) or ground state bleach (GSB at $\lambda<390 \mathrm{~nm}$ for NAIP and $\lambda<430 \mathrm{~nm}$ for MeONAIP). Positive $\triangle A$ signals are coded in red and assigned to excited state absorption (ESA, at early times in the range $400-450 \mathrm{~nm}$ for both compounds) or photoproduct absorption (PA, t $>0.2$ ps and $\lambda>380$ $\mathrm{nm}$ for NAIP and $\mathrm{t}>0.3 \mathrm{ps}, \lambda>420 \mathrm{~nm}$ for MeO-NAIP).

Figure 2 compares an overview of the TA data of methanol solutions of Z-NAIP and Z-MeO-NAIP upon $400 \mathrm{~nm}$ excitation. Despite a 30-nm blue-shift of the ground state absorption of the former (see Figure $1 \mathrm{~B})$, both molecules display similar transient spectra at early times $(<0.2 \mathrm{ps})$, with excited state absorption (ESA) and stimulated emission (SE) at wavelengths $<450 \mathrm{~nm}$ and $>450 \mathrm{~nm}$, respectively. In the following we describe specifically the photoisomerization dynamics of NAIP, which is very similar and representative of the behavior of Z-ZW-MeO-NAIP [24] and of both isomers of MeO-NAIP [21, 23]. A characteristic feature of the NAIP TA data is the spectrally very broad SE band extending to wavelengths $>650 \mathrm{~nm}$, which decays on a time scale shorter than $200 \mathrm{fs}$ and is followed by a weak, positive, equally broad absorption band, as best seen in Figure 3A. The positive signal after $200 \mathrm{fs}$ is attributed to the earliest signature of the photoproduct, by analogy with the case of ZW-MeO NAIP where the same signatures are observed and time-resolved Mid-IR spectroscopy showed in addition that the central $C=C$ is reconstituted - indicating the decay to the ground state - on the same time scale. [24] Figure $3 A$ clearly reveals that with increasing probing wavelengths the zero-crossing between the negative SE and positive PA signals occurs at later time delays while the maximum of the PA signal is reached at earlier times (see arrows in Figure3A). This temporal behavior is assigned to the signature of a vibrational wavepacket evolving along the reaction coordinate on the $S_{1}$ PES towards the CInt (red shift of the SE), decaying to $S_{0}$ via the CInt, and further evolving towards the $S_{0}$ minimum (blue shift of the PA). A similar TA signature was also observed in Rhodopsin [25] and other IP compounds [21, 24] and interpreted the same way. 

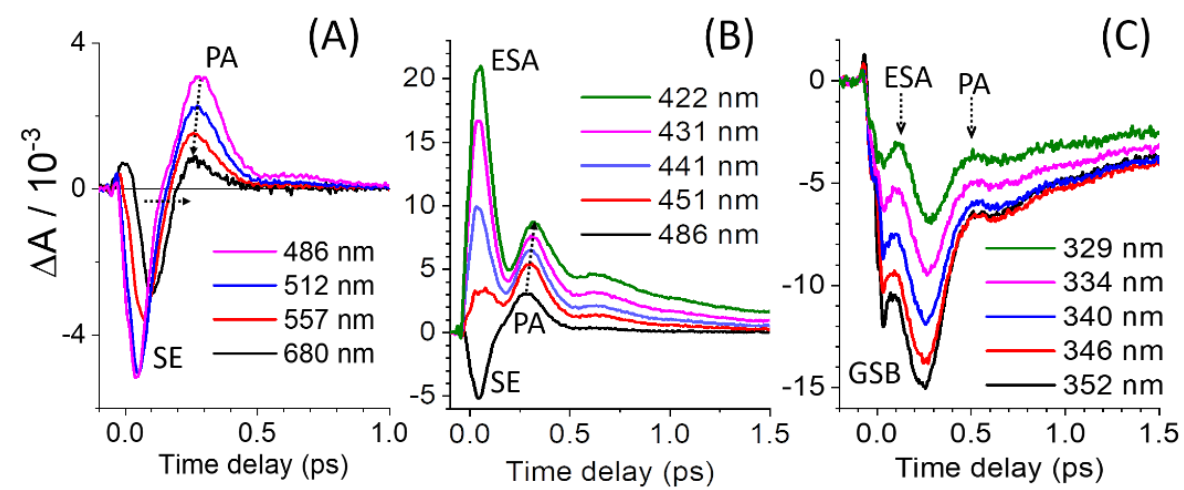

Figure 3: TA kinetic traces at a selection of probing wavelengths for NAIP in methanol upon $\mathbf{4 0 0} \mathbf{n m}$ excitation. Illustration of (A) the rapid red shift of the SE and blue shift of the PA in the red part of the observation window (> $486 \mathrm{~nm}$ ), (B) the short-lived $\mathrm{S}_{1}$ state signatures (SE and ESA) followed by the abrupt rise and further blue shift of the PA signature, after $300 \mathrm{fs}$, in the $420-490 \mathrm{~nm}$ probing window, (C) the dynamic blue shift and decay of the delayed ESA signature overlapping the GSB signal, followed by the sudden recovery of the GSB and PA signal onset, between 400 and $500 \mathrm{fs}$, in the 330 $-350 \mathrm{~nm}$ spectral region.

After its decay at the CInt, the ground state evolution of the vibrational wavepacket can be monitored by tracking temporally and spectrally the early PA signature. As discussed above, it suddenly rises with a non-exponential kinetics as already observed in the case Z-ZW-MeO-NAIP [24] - after $250 \mathrm{fs}$ in the 500 to $700 \mathrm{~nm}$ detection range (Figure $3 \mathrm{~A}$ ). After $\sim 300 \mathrm{fs}$, the PA signal is seen to rise abruptly in the 420 - $500 \mathrm{~nm}$ detection window (Figure 3B, after the decay of the early SE and ESA signatures). Finally, the PA signature appears after $\sim 400$ to $500 \mathrm{fs}$ in the 320 - $350 \mathrm{~nm}$ range (Figure $3 \mathrm{C}$ ), causing the sudden ground state bleach (GSB) recovery. Hence, the PA signal undergoes a very large, dynamic blue shift over the entire UV-Vis spectrum between 250 and $500 \mathrm{fs}$. This is interpreted as the signature of the wavepacket rapidly evolving from the Clnt region towards and over the photoproduct $\mathrm{S}_{0}$ energy minimum. Like in other IP compounds [21, 24], subsequent low-frequency oscillations are observed in the range $320-450 \mathrm{~nm}$ as illustrated in Figure 4 . The inset of Figure 4 displays the oscillatory residuals of a multiexponential fit (solid green lines) together with the kinetic trace observed at $680 \mathrm{~nm}$. Again, the latter shows the earliest, red-shifted, $\mathrm{S}_{0}$ signature of the wavepacket, soon after passing through the CInt (arrow number 1). Then, arrows number 2 and 3 in Figure 4 pinpoint the first passage of the wavepacket through different spectral observation windows around $425 \mathrm{~nm}$ and $325 \mathrm{~nm}$ successively. Subsequent oscillations are clearly distinguishable from the noise, and appear out-of-phase in the 425 $\mathrm{nm}$ and $335 \mathrm{~nm}$ traces which are respectively in the red and blue sides of the PA absorption spectrum. This is reminiscent of similar, low-frequency oscillations observed in Rhodopsin[11], and is indicative of further oscillatory motion of the wavepacket around its equilibrium position in the ground state. Fourier transform of both residuals yields a vibrational period of $400+/-50 \mathrm{fs}\left(\sim 80 \mathrm{~cm}^{-1}\right)$. Most importantly, when performing the same TA experiment on the parent MeO-NAIP, with much shorter $(<8 \mathrm{fs})$ resonant $(400 \mathrm{~nm})$ or non-resonant $(800 \mathrm{~nm})$ pump pulses, we recently showed, [23] that these ground state oscillatory motion is not produced by a so-called Resonant Impulsive Stimulated Raman process induced by the pump pulse in $\mathrm{S}_{0}$ [41-43]. Instead they must be attributed to a vibrationally coherent motion initially triggered on $\mathrm{S}_{1}$ along the reaction coordinate, by the steep potential energy surface. The $80 \mathrm{~cm}^{-1}$ mode is assigned to the reaction coordinate comprising the $\mathrm{C1}^{\prime}=\mathrm{C} 4$ bond twist coupled to the 5-membered rings deformations.[21] The vibrational coherence is preserved upon 
decay at the CInt, and observed on the So PES where it damps with a sub 300 -fs time constant - as in other IP compounds [21] - corresponding to the vibrational dephasing time scale.

In all IP compounds, the $E$ isomer absorption maximum is blue shifted with respect to the $Z$ isomer, by no more than 3 to $8 \mathrm{~nm}$ (see e.g. Figure 1C). Therefore, it is actually not possible to conclude from the above data whether the early PA signature corresponds to a vibrational wavepacket forming the $E$ isomer (successful isomerization) or the $Z$ isomer (aborted isomerization reforming the reactant). As we will discuss in Section $V$, the photoisomerization quantum yield for NAIP is $\sim 20 \%$, meaning that the abortion channel dominates. Hence, we likely observe a vibrationally coherent reformation of the reactant. Still, it remains that the vibrationally coherent reactive motion in $S_{1}$ drives the systems through the Clnt to $S_{0}$ on a time scale faster than vibrational dephasing. In a purely quantum mechanical, coherent picture, the $S_{1}$ vibrational wavepacket splits at the Clnt in a coherent superposition of $E$ and $Z$ components, and rapid decoherence turns this coherent superposition into a statistical ensemble which further relaxes to a final proportion of $20 \% E$ and $80 \% Z$.

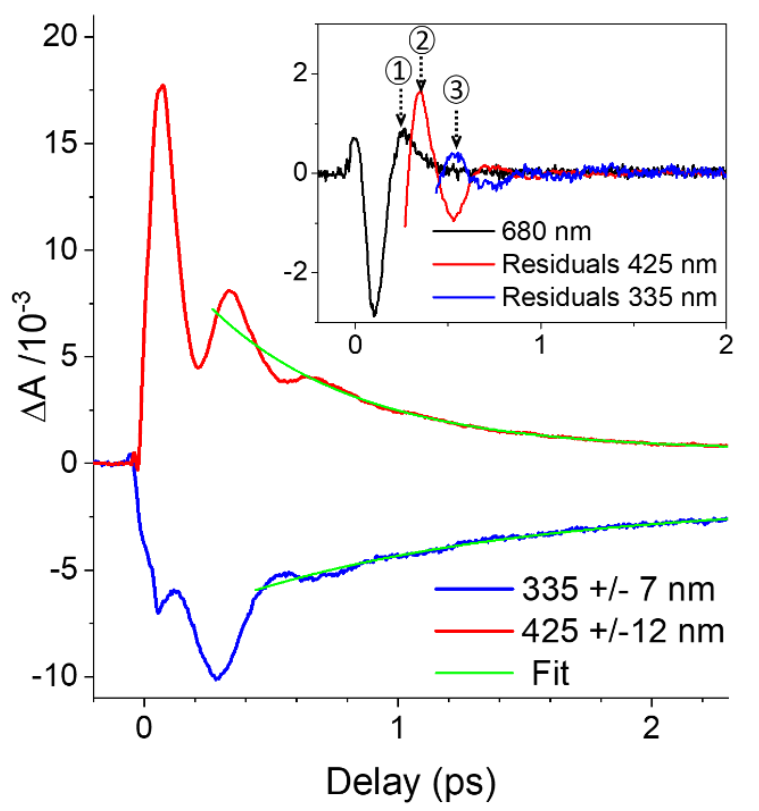

Figure 4: Oscillatory TA signal observed in NAIP after decay to the ground state. Inset: The residuals of the (multi)exponential fit (green lines) exhibit oscillations which are compared to the transient absorption signal observed at $680 \mathrm{~nm}$.

In the red part of the spectrum, the sudden crossover between the SE and the PA signals occurs at $\sim 190 \pm 30 \mathrm{fs}$ for Z-NAIP (see Figure 3a, $680 \mathrm{~nm}$ kinetics). This is an estimate of the moment at which the vibrational wave packet decays to $S_{0}$ through the $C$ Int. The same peculiar signature is observed in Z- MeO-NAIP and Z-ZW-MeO-NAIP, where the passage through the CInt is estimated in the same way to be $260 \mathrm{fs}$ and $270 \mathrm{fs}$, respectively. Also in Rho, the decay to the ground state estimated from a similar SE to PA crossover occurring after $\sim 75 \mathrm{fs}$. [25]

In the following, we will define as scenario I, the photoisomerization of all IP compounds which display this SE/PA signal crossover, interpreted as a characteristic signature of the vibrationally coherent reactive motion through the $\mathrm{Clnt}$. Within this set of compounds, we may however distinguish different 
degrees of vibrational coherence. In Z-NAIP (Figures 4) as well as Z-ZW-MeO-NAIP [24] and both isomers of MeO-NAIP, [21] the SE/PA signal crossover is followed by pronounced, reaction-induced, $\mathrm{S}_{0}$ oscillations along the low-frequency, torsion mode of the reaction coordinate. These compounds are characterized by a "high degree" of vibrational coherence. They undergo what we call a biomimetic photoisomerization, in the sense that they reproduce vibrational coherence signatures similar to Rho. By contrast, E-ZW-MeO-NAIP [44] displays the SE/PA signal crossover assigned to the vibrationally coherent early motion of scenario $\mathrm{I}$, but no detectable signatures of subsequent $\mathrm{S}_{0}$ oscillatory motion, thus displaying a lower or intermediate degree of vibrational coherence.

\section{Photoisomerization scenario II illustrated with Ch-MeO-NAIP.}

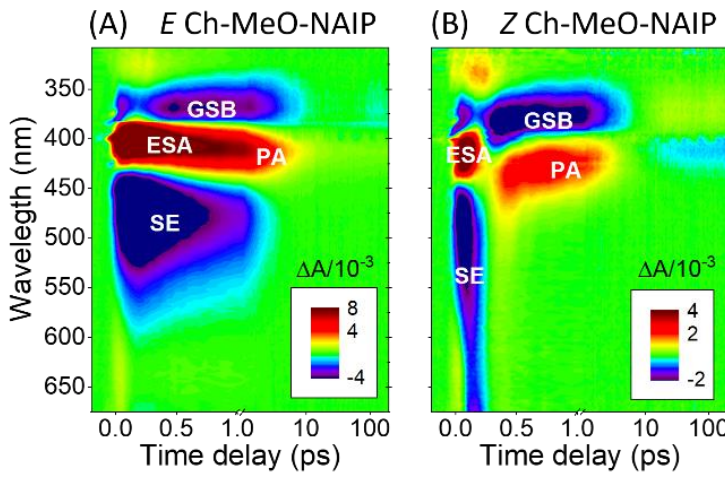

(C) Spectral integration over $460-560 \mathrm{~nm}$

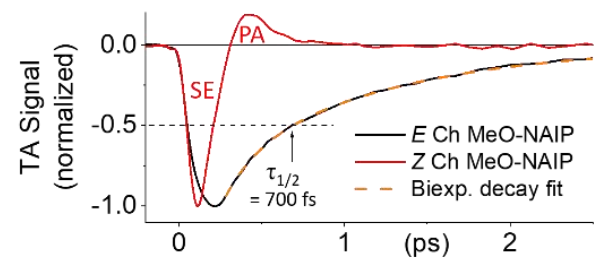

Figure 5: Transient absorption ( $\triangle A$ ) 2D maps of $(A)$ the pure $E$ and $(B)$ the pure $Z$ isomers of Ch-MeONAIP in MeOH upon $\mathbf{4 0 0 ~} \mathbf{n m}$ excitation, plotted as a function of probe wavelength $(\mathrm{nm})$ and time delay (ps) between pump and probe pulses. Note the break at 1 ps between linear and logarithmic time scales. (C) TA kinetic traces of $E$ - and $Z$ - isomers, obtained by integrating the 2D maps over the 460 $560 \mathrm{~nm}$ probing window. For the Z-isomer the SE signal decays abruptly and is followed by the PA signal. For the $E$-isomer instead, the $S E$ signal is seen to rise until $250 \mathrm{fs}$, and its decay - beyond 300 fs - can be fitted by a bi-exponential function (orange dashed line; see result in Table 2). The lifetime of the corresponding transient bright $S_{1}$ state may also be estimated by its half-life $\tau_{1 / 2}=700 \mathrm{fs}$.

In contrast to scenario I illustrated above, we observe a qualitatively distinct scenario - referred to as scenario II - for both isomers of the $\mathrm{C} 5$ non-methylated dMe-MeO NAIP $[23,36]$ and its chiral analogue Ch-dMe-MeO-NAIP.[34] Here, we illustrate the difference between the two scenarios with the example of another compound, the Ch-MeO-NAIP (i.e. compound 7b of ref [17]). Its TA data are displayed for the first time in Figure 5 for the pure $E$ - and $Z$-isomers. The two data sets are reconstructed by computing the appropriate linear combinations (see Section II and Figure S1 of the SI) of the TA data recorded on DS and PSS samples with E:Z compositions of $99 \%: 1 \%$ and $30 \%: 70 \%$, respectively (see Table 1 ). Noticeably, the $E$-isomer has a photoisomerization scenario similar to that 
of the C5-non-methylated compounds. [23, 34] In particular, its stimulated emission is spectrally narrower and much longer-lived (see Figure 5A), as compared to that of the Z-isomer (Figure 5B) where we recognize instead the signatures of a vibrational wavepacket travelling through the CInt - i.e. the SE/PA crossover discussed above - with a zero-crossing between SE and PA signals occurring at $280 \mathrm{fs}$ in the red-most part of the observation window (i.e. at $680 \mathrm{~nm}$ probing wavelength, see Figure S4 of the $\mathrm{SI}$ ). Figure $5 \mathrm{C}$ illustrates the major spectroscopic difference between the Vis signatures of the $\mathrm{S}_{1}$ state of both isomers. For the $E$-isomer, the negative $S E$ signal is seen to rise on a time scale slower than the experimental time resolution, up to $\sim 250 \mathrm{fs}$ (Figure 5C, black curve). This indicates relaxation away from the initial Franck Condon region to form, on this time scale, a transient, bright conformation emitting at $\lambda_{S E} \sim 480 \mathrm{~nm}$. Beyond this initial, non-exponential kinetics we can fit the SE decay by a biexponential decay function (dashed orange line) with time constants $190 \mathrm{fs}(55 \%)$ and $1.1 \mathrm{ps}(45 \%)$. This overall kinetics of SE signal can also be characterized by its half-life of $690 \pm 20 \mathrm{fs}$, which may be considered as a measure of the typical lag time of the $S_{1}$ population in this "bright" state. We note, that similar SE bands and non-exponential SE kinetics are also observed for both isomers of the C5non-methylated compounds (see Figure S2 and S3 of the SI). For E-Ch-MeO-NAIP (Figure 5A), the PA band ( $\mathrm{S}_{0}$ signature) is observed almost at the same wavelength as the ESA ( $\mathrm{S}_{1}$ signature), which prevents resolving the kinetics for the PA signal rise. Interestingly, this is not the case for the C5 nonmethylated compounds, where the PA formation and ESA decay kinetics are observed at slightly distinct wavelengths (see e.g. Figure S2 of the SI). For the Ch-dMe-MeO-NAIP in particular, [34] the PA formation rate coincides with the SE or ESA decay rates. This scenario is in marked contrast with the abrupt SE decay and delayed PA rise of scenario I. However, we note that the half-life of the IP compounds obeying scenario II is sub-picosecond (see Table 2). This remains significantly faster than vibrational thermalization, which is estimated to occur with a time constant of $5 \mathrm{ps}$ in the $\mathrm{S}_{0}$ state of the IP compounds, $[21,24]$ or $4 \mathrm{ps}$ in the $\mathrm{S}_{1}$ state of related, retinal-inspired compounds showing longer excited state lifetimes. [30] Hence scenario II is an intermediate regime between the vibrationally coherent scenario I and a fully stochastic, thermally-equilibrated photo-isomerization scenario.

\section{Discussion and conclusion}

\section{a) Interpretation of the TA data kinetics}

The photoisomerization dynamics of all IP compounds studied experimentally so far can be categorized in the two distinct scenarios illustrated in Figure 6 . In the vibrationally coherent scenario I, the photoreaction is faster than vibrational dephasing, as evidenced by the observation of the $300-\mathrm{fs}$ damped oscillatory signal in the photoproduct electronic ground state. This is due to the fact that the population is rapidly accelerated away from the FC region and undergoes a nearly ballistic motion until the photoproduct formation, with no significant lag time in any intermediate state, such that one can follow the gradual evolution of the spectroscopic signature of this population all along the reactive path through the CInt until the PA (Figure 6A). In scenario II instead (Figure 6B), the fast early motion out of the FC does not launch a ballistic motion towards the Clnt, but rather results in the formation of a transient bright state. The lag time of the population in this state - estimated by the SE signal half- 
life - is much longer than the actual evolution time through the CInt, such that the population of transient configurations in between the bright state and the $\mathrm{S}_{0}$ final state remains at any time very low and not detectable. This explains why the PA signal appears to rise concomitantly with the SE decay,[34] and not subsequently in the 700-nm range like in scenario I.

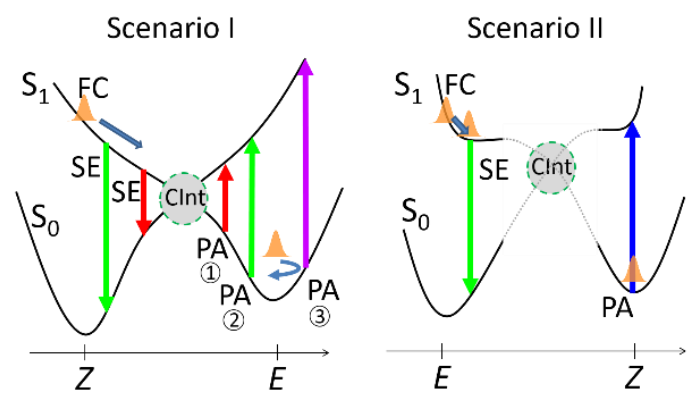

Figure 6: Schematic illustration of photoisomerization scenarios I (A) and II (B) of the IP photoswitches. In scenario $\mathrm{I}$, the $\mathrm{S}_{1}$ population is strongly accelerated along the steepest PES gradient and its UV-Vis TA signature can be followed progressively along the reactive motion through the CInt until the photoproduct formation. The numbers $1,2,3$ denote the sequential detection of the $S_{0}$ vibrational wavepacket in the successive spectroscopic windows, as discussed in the insert of Figure 4 for the case of NAIP. In scenario II, following a fast relaxation from the FC region, the population reaches in a bright state for a lag time much longer than the time scale for transiting through the Clnt such that no significant transient population is detectable in the dashed zone of the PES's, but only the final PA signal, formed on a time scale slower than vibrational dephasing.

\section{b) The molecular origin of the vibrational coherence in the IP compounds}
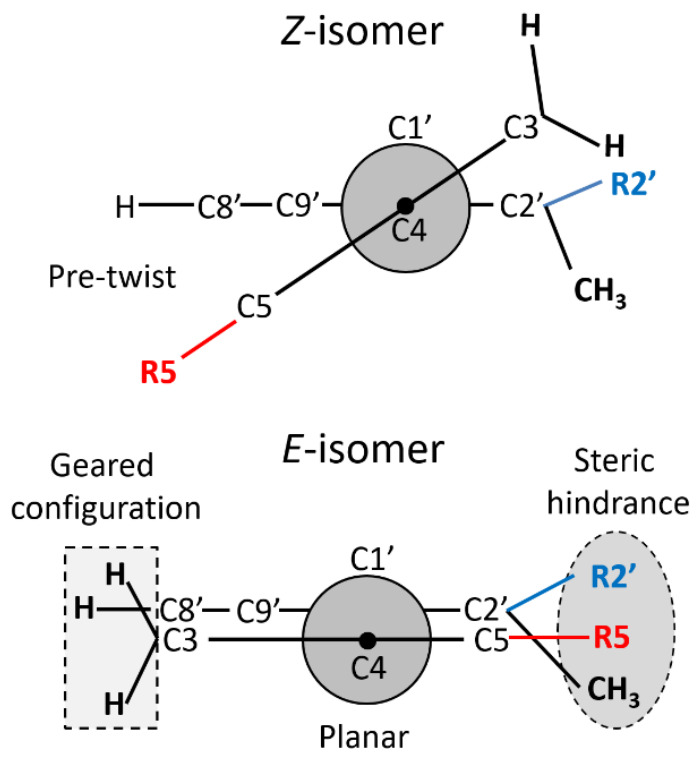

Figure 7: Newman projections of both isomers of the IP compounds: $R 2^{\prime}=H=$ chiral; $R 2^{\prime}=C_{3}=$ achiral; $\mathrm{R} 5=\mathrm{H}=$ "demethylated"; $\mathrm{R} 5=\mathrm{CH}_{3}$ otherwise. Top: Z-isomer adopting a pre-twisted conformation; Bottom: $E$-isomer in a "geared", planar conformation. If $\mathrm{R} 5=\mathrm{CH}_{3}$, the steric clash with the substituents of $\mathrm{C}^{\prime}$ ' destabilizes the $E$ configuration, such that the $Z$ isomer becomes more stable, 
although it adopts a twisted conformation due to the residual steric hindrance between $\mathrm{R} 5$ and $\mathrm{H}-\mathrm{C} 8$ ', and between the substituents of $\mathrm{C} 2^{\prime}$ and $\mathrm{C} 3$.

After illustrating the spectroscopic signatures of both photoisomerization scenarios, we now discuss their mechanistic origin. To this end, it will be useful to consider Figure 7 displaying the Newman projections of both IP isomers in $\mathrm{S}_{0}$. In our previous comparison of Z-MeO-NAIP and E-dMe-MeO-NAIP, [23] we reported that the nature of the group on $\mathrm{C} 5$, i.e. $\mathrm{R} 5=\mathrm{H}$ or $\mathrm{CH}_{3}$ in Figure 7, controls the relative stability and planarity of the isomers. In addition, based on computational investigations, we argued, that the steric effects result in an electronic effect which in turn controls the slope of the $\mathrm{S}_{1}$ potential energy surface and the photoisomerization dynamics. More specifically we predicted that in a planar geometry (E-dMe-MeO-NAIP), the reactive $S_{1}$ and non-reactive $S_{2}$ electronic states are nearly degenerate and their mixing increases the bonding character $-i$. e. reduces the reactivity - of the isomerizing $\mathrm{C}=\mathrm{C}$ bond. [45] As a result, the molecular wavepacket evolves on a rather flat $\mathrm{PES}$ resulting in a slower evolution towards the CInt and a loss of vibrational coherence by the time the ground state photoproduct is detected. This would explain the above scenario II. Instead, an $\mathrm{S}_{0}$ pre-twist of the isomerizing $\mathrm{C}=\mathrm{C}$ bond - induced when $\mathrm{R} 5$ is $\mathrm{CH}_{3}$ in the parent Z-MeO-NAIP - lifts the $\mathrm{S}_{1}-\mathrm{S}_{2}$ degeneracy, thus producing a much steeper $S_{1} P E S$, which strongly accelerates the wavepacket towards the CInt. This explains the photoisomerization scenario I, as depicted on Figure 6A.

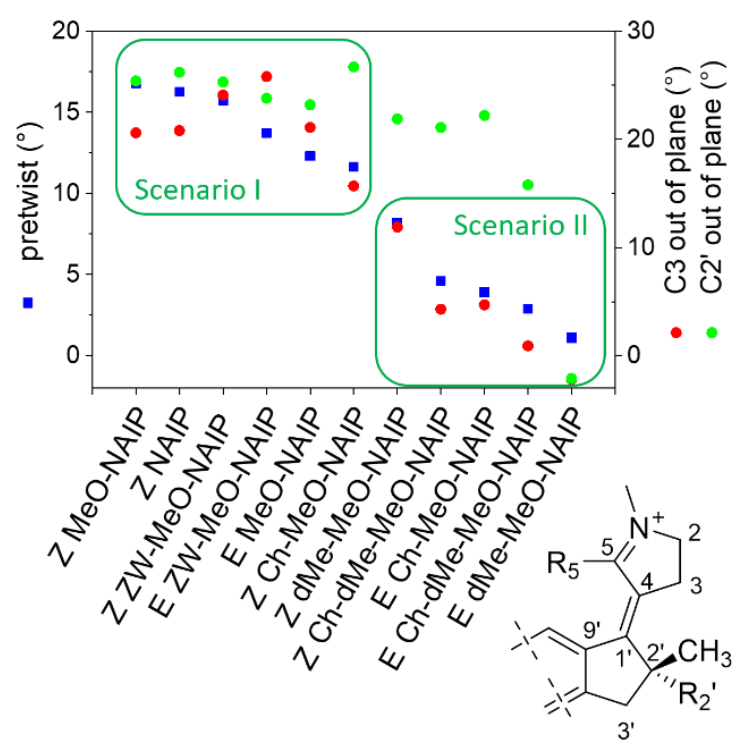

Figure 8: Correlation between $S_{0}$ equilibrium structures and the observation of vibrational coherence. We define the double bond pre-twist angle (blue squares) as the average of the $\mathrm{C} 9^{\prime}-\mathrm{C} 1^{\prime}=\mathrm{C} 4$ $\mathrm{C} 5$ and $C 2^{\prime}-\mathrm{C1}^{\prime}=\mathrm{C} 4-\mathrm{C} 3$ dihedral angles. We also report the predicted out-of-plane distortions of $\mathrm{C} 3$ (red dots) and C2' (green dots), given by the C5-C4-C3-C2 and C9'-C1'-C2'-C3' dihedral angles, respectively.

Here we tentatively generalize to all IP compounds the correlation between the observation of a vibrationally coherent photoisomerization and the deformation away from planarity of the $S_{0}$ equilibrium structure. To this end, we model the $S_{0}$ structure of all IP's, at the DFT level of theory including implicit solvent correction (using a polarizable solvent model, see Section II) and report on Figure 8 the values of three specific dihedral angles for all IP compounds. One of the three angles documents the $\mathrm{C}=\mathrm{C}$ double bond pre-twist discussed in ref [23]. The other two quantify out-of-plane distortions of $\mathrm{C} 3$ and $\mathrm{C2}$. The pre-twist of the Z-MeO-NAIP and E-dMe-MeO-NAIP are $17^{\circ}$ and $1^{\circ}$, 
respectively, in good agreement with the results of previous, electronic structure modeling of the same compounds with implicit solvent [36] $\left(18^{\circ}\right.$ and $\left.2^{\circ}\right)$ or explicit solvent (i.e. using a box of solvent molecules as part of a QM/MM model of the solvated IP's) at ambient temperature [23] $\left(15^{\circ}\right.$ and $\left.3^{\circ}\right)$.

Noticeably, the dihedral angles corresponding to the pre-twist and the out-of-plane distortions of the C3 atom are correlated and adopt values which exceed $10^{\circ}$ and $15^{\circ}$ respectively for all compounds which obey photoisomerization scenario I, while the same angles remain below $8^{\circ}$ and $12^{\circ}$ respectively for all compounds obeying scenario II. Instead, no apparent correlation exists between the C2' out-ofplane displacement and the photoisomerization scenario. Within the compounds obeying scenario I, we observe that those which have the strongest $\mathrm{S}_{0}$ pre-twist - that is $>15^{\circ}$ like Z-MeO-NAIP, Z-NAIP and Z-ZW-MeO-NAIP, see Figure 8 - are also characterized by the highest degree of vibrational coherence, i.e. display the most pronounced reaction-induced, ground state oscillatory signals.

The present work supports the generalization of our previous result [23] reporting a correlation between the vibrational coherence of the photoisomerization and the ground state distortion from planarity. More specifically, we conclude that the steric hindrance leading to out-of-plane distortion of the $S_{0}$ equilibrium structure is (i) the manifestation of a strain (a force) acting along the twisting angle of the reactive $\mathrm{C}=\mathrm{C}$ bond in both $\mathrm{S}_{0}$ and $\mathrm{S}_{1}$ states, which is released when the same $\mathrm{C}=\mathrm{C}$ bond is unlocked by the electronic excitation. It also results (ii) in an increase of the $\mathrm{S}_{1}-\mathrm{S}_{2}$ energy gap and, therefore, to a decrease in the mixing of $S_{1}$ with the non-reactive $S_{2}$ state. Both (i) and (ii) effects lead to a steeper $S_{1}$ PES which accelerates the system towards the CInt and favors the onset of vibrational coherence in the IP's.

\section{c) Excited State Life time (ESL) determination}

The excited state lifetimes (ESLS) of all compounds are reported in Table 2. Here, it is important to notice, that the definition of the ESL is not the same in both photoisomerization scenarios. Indeed, in scenario I, the signature of the vibrational wavepacket crossing the CInt allows us to define the ESL as the moment at which the entire population - or most of the population - decays from $S_{1}$ to $S_{0}$. In scenario II, however, the ESL is instead estimated from the decay kinetics of the stimulated emission signal, which is not well fitted by a usual multiexponential function, due to the initial non-exponential rise of the signal. Only after this rise, i.e. beyond 250 to $300 \mathrm{fs}$, it is possible to fit the SE kinetics using a bi-exponential decay. Therefore, we propose that the half-life of the SE signal is a better estimate of the $S_{1}$ bright state lifetime (see Figure S3 in the SI). Notice that, in scenario I a biphasic SE decay may not be observed since the PA signal abruptly rises at the same wavelengths, thus overlapping and masking any putative longer-lived SE compound. However, time-resolved fluorescence spectroscopy performed using the up-conversion technique - also revealed a biphasic fluorescence emission for the vibrationally coherent MeO-NAIP [19] and ZW-MeO-NAIP [24]. Hence we report in Table 2 the fitting parameters of the bi-exponential decay of the stimulated or fluorescence emission, and define the ESL of compounds obeying scenario II as the SE half-life, as defined in Figure 5C (see also Figure S3 in the $\mathrm{SI})$.

Table 2: Comparison of the photoisomerization dynamics properties of the IP compounds. (a) Biexponential fit of the femtosecond fluorescence decay (up-conversion set-up) for scenario I, or of the 
SE signal for scenario II. (b) Excited state Life Time (ESL) estimated either $\left({ }^{*}\right)$ as the time of crossover between SE and PA signatures as observed in the red-most part of the probing window (i.e. $>680 \mathrm{~nm}$, see SI Figure S4) for scenario I, or (**) as the half-life of the bright $\mathrm{S}_{1}$ state for scenario II (see SI, Figure S3). (c) Photoisomerization Quantum yields determined within $+/-2 \%$, according to the procedure described in Section II. (d) Independent determinations of the QYs from previous reports.

\begin{tabular}{|c|c|c|c|c|c|}
\hline $\begin{array}{c}\text { Photo- } \\
\text { isomerization } \\
\text { scenario }\end{array}$ & Compound & $\begin{array}{l}\text { Fluorescence [Ref] or SE bi- } \\
\text { exponential decay kinetics (a) }\end{array}$ & $\begin{array}{c}\mathrm{ESL}(\mathrm{b}) \\
(\mathrm{fs})\end{array}$ & $\begin{array}{l}\text { QY } \\
\text { (c) }\end{array}$ & $\begin{array}{l}\text { QY (b) } \\
\text { [Ref] }\end{array}$ \\
\hline \multirow[t]{6}{*}{ Scenario I } & Z-NAIP & -- & $190^{*,(i)}$ & 21 & -- \\
\hline & Z-MeO-NAIP & $<40 \mathrm{fs}(-) ; 300 \mathrm{fs}(-)$ [19] & $260 *$,(i),(ii) & $21 t$ & $21[18,19]$ \\
\hline & E-MeO-NAIP & -- & $270^{* \text {,(ii) }}$ & -- & 34 [18] \\
\hline & Z-ZW-MeO-NAIP & 140 fs (87\%); 1 ps (13\%) [24] & $270^{* \text { (iii) }}$ & 32 & $35[24]$ \\
\hline & E-ZW-MeO-NAIP & -- & $350 *$,(iii) & 15 & $15[44]$ \\
\hline & Z-Ch-MeO-NAIP & -- & $280^{*,(i)}$ & 13 & -- \\
\hline \multirow[t]{5}{*}{ Scenario II } & E-Ch-MeO-NAIP & 190 fs (55\%) ; 1.1 ps (45\%) & $690 * *$,(iv) & 18 & -- \\
\hline & Z-dMe-MeO-NAIP & 210 fs $(80 \%) ; 0.5$ ps (20\%) & $430 * *$,(iv) & 24 & -- \\
\hline & E-dMe-MeO-NAIP & 260 fs $(90 \%) ; 0.8$ ps (10\%) & $480 * *$,(iv) & 26 & $25[36]$ \\
\hline & Z-Ch-dMe-MeO-NAIP & 260 fs (55\%); 1.2 ps (45\%) & $830 * *$,(iv) & 20 & -- \\
\hline & E-Ch-dMe-MeO-NAIP & 350 fs (50\%); 1.3 ps (50\%) & $830 * *$,(iv) & 24 & -- \\
\hline
\end{tabular}

(i) This work, see SI Figure S4; (ii) TA data from ref [21]; (iii) TA data from ref [44]; (iv) This work, see SI, Figure S3.

${ }^{+}$The $21 \%$ value for the QY of Z-MeO-NAIP is taken from ref $[18,19]$ and used as a reference to calibrate all other QY values (see Section II).

From the data collected in Table 2, it appears that the photoisomerization quantum yield does not correlate with the ESL. This holds for both photoisomerization scenarios, and is in contrast with early expectations based on the Landau-Zener model for non-adiabatic transitions, [4, 46] but also with the anti-correlation reported for PSBR derivatives in solution. $[47,48]$ We argue here, that such a correlation may, a priori, not be expected because the quoted ESL is actually the time scale for the SE signal decay - i.e. for the motion away from the region of the conformational space which has a significant $\mathrm{S}_{0}-\mathrm{S}_{1}$ optical transition probability - while the photoisomerization $\mathrm{QY}$ is presumably ruled by the precise reactive motion in the vicinity of the $\mathrm{Clnt}$, which is a distinct region of the conformational space.[49] Moreover, the recent investigations of the Rho photoisomerization dynamics [14, 16] support a mechanism where the QY is controlled by the vibrational phase of the HOOP mode at the point of decay from $S_{1}$ to $S_{0}$ (i.e. near the $\mathrm{Clnt}$ ). This is fundamentally different from the semi-classical Landau-Zener picture predicting correlation between reaction speed and QY.

Hypothetically, the change of electronic structure occurring at the Clnt upon decay from $S_{1}$ to $S_{0}-$ i.e. the reformation of the central double bond upon restoring the p-orbital overlap of both carbon atoms (C1' and C4 in IP) - would be modulated by the C2' and C3 out-of-plane (COOP) motions in the IP, exactly like it is modulated by the HOOP mode of the reactive $\mathrm{HC} 11=\mathrm{C} 12 \mathrm{H}$ moiety in Rho (see Figure 9 $A$ and $B$ ). [16] In simple wording, when, at decay, the HOOP is in a phase corresponding to a $\mathrm{H}$ motion relocating the two hydrogens in their original reactant position - i.e. the 11-cis position - then the decay leads to an unsuccessful event meaning that the reaction is aborted (see Figure $9 \mathrm{C}$ ). In contrast, if the phase is such that the H's are moving in the opposite direction (i.e. towards the all-trans 
conformation characteristic of the photoproduct, see Figure 9D), then the decay is successful.[16] In the vibrationally coherent photoisomerization of Rho, the HOOP oscillates typically 3 times before the system arrives at the CInt. By that time, little vibrational dephasing has occurred, thus, in principle, the fine tuning of the PES allows modifying the oscillation phase of the entire population, and therefore significant QY tuning is possible. This leads us to hypothesize that Rho enhances the PSBR QY by tuning its PES and taking advantage of the vibrational coherence. Because of the direct analogy between the role of HOOP in PSBR and COOP in IP at modulating the p-orbital overlaps (Figure 9 C, D), we expect a similar picture to hold also for the vibrationally coherent IP compounds. However, the high-frequency HOOP mode of Rho is replaced by a lower-frequency carbon out-of-plane (COOP) motions in IP. Hence, by the time the IP's reach the CInt and decay, the phase of the COOP mode may not change significantly within this set of compounds. We hypothesize, that the CInt is reached with a COOP velocity - and therefore a phase - mostly resulting in abortion rather than completion of the isomerization reaction, which would explain why the QY remains in the $25+/-10 \%$ range in the present set of compounds.
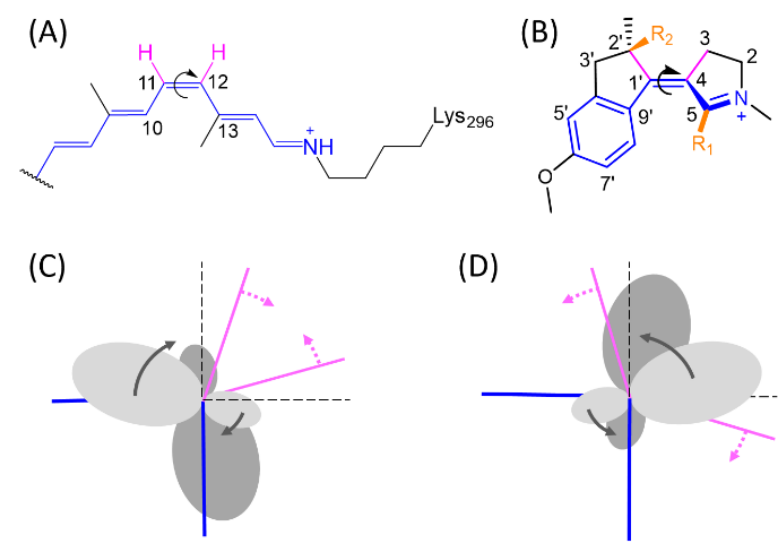

Figure 9: Analogy between Rho and IP photoisomerization mechanism in the vicinity of the Clnt. The Hydrogen-Out-Of-Plane (HOOP, purple bonds) motion in Rho (A) and Carbon-Out-Of-Plane (COOP, purple bonds) motion in NAIP (B) have the same effect of pyramidalizing the carbon atoms of the isomerizing double bonds and thus controlling their p-orbital overlap. Panel (C) and (D): Newman projections along the isomerizing bond at the CInt, i.e. $90^{\circ}$ torsion angle between the blue bonds representing the carbon backbones of either Rho or NAIP. Depending on whether the angle between both purple bonds reduces (C) or increases (D), then the p-orbitals (grey lobs) overlap is restored so as to abort or complete the torsion motion, respectively.

\section{Conclusion}

We presented a comprehensive experimental TA investigation of a family of IP compounds. Two distinct photoisomerization scenarios are observed. The first one is a so-called vibrationally coherent photoisomerization. It is observed for IP compounds significantly distorted from planarity (pretwist > $10^{\circ}$ ) in their ground state, and is attributed to a strong acceleration of the $\mathrm{S}_{1}$ population along the reaction coordinate towards and through the CInt, due to a steep PES. As a result, the photoisomerization occurs on a time scale faster than vibrational dephasing, and for the compounds having the strongest pretwist $\left(>15^{\circ}\right)$ this results in the observation of an oscillating wavepacket in the photoproduct. The second scenario describes sub-picosecond photoisomerizations occurring in an intermediate regime between the vibrationally coherent scenario I and a thermally-equilibrated, 
stochastic photoisomerization. It is observed in compounds which are less distorted from planarity. For these compounds, a transient bright excited state is populated over a significant lag time during which vibrational dephasing already occurs before the decay event at the Clnt. Computational investigations indicate that for these compounds, the $S_{1}$ PES is flatter, due to the proximity and mixing of $S_{1}$ with $S_{2}$.

A major target of this investigation is to understand the mechanism which defines the photoisomerization quantum yield (QY) of these compounds. An accurate comparison of the $Q Y$ ' $s$ of the IP compounds does however not reveal any correlation between the $Q Y$ and the photoisomerization dynamics, characterized by their vibrational coherence character and their excited state lifetimes. The analogy between PSBR and IP reaction coordinates suggest that the COOP mode of IP plays a role similar to that of the HOOP mode of PSBR, at controlling the $\mathrm{C}=\mathrm{C}$ double bond reconstitution at the decay event, in particular for the vibrationally coherent compounds. The small variability of the QY's of all IP compounds (25+/- $10 \%$ ) may result from the fact that the COOP mode frequency is too low in order to actively tune the QY within the present set of compounds. We hypothesize that this lack of enhancement of the $Q Y$ in the present set of vibrationally coherent compounds (scenario I) is related to our lack of understanding of the phase relationship between the vibrational modes of the substituents at the reactive $C=C$ bond - analogues of the HOOP mode ( 900 $\mathrm{cm}^{-1}$ ) controlling the PSBR QY in Rho - and the isomerization coordinate of IP. Further investigations are necessary to understand the key parameters controlling the dynamics and efficiency of $\mathrm{C}=\mathrm{C}$ double bond photoisomerization, a target of central relevance to enable the rational design of efficient molecular switches and devices.

\section{Acknowledgements}

We acknowledge support from the ANR program "Investissement d'Avenir", via grants ANR-10-IDEX0002-02, ANR-11-LABX-0058_NIE, ANR-10-LABX-0026_CSC, ANR-10-EQPX-52-01, and from the Region Alsace (Contrat doctoral, No. 607-12-C31). M.P. and M.O. are grateful for the Italian MIUR, PRIN 2015 grant and for a Department of Excellence grant 2018-2022.

\section{REFERENCES}

1. Koumura, N., Zijlstra, R. W. J., van Delden, R. A., Harada, N., \& Feringa, B. L. (1999). Light-driven monodirectional molecular rotor. Nature, 401, 152. doi:10.1038/43646

2. Roke, D., Wezenberg, S. J., \& Feringa, B. L. (2018). Molecular rotary motors: Unidirectional motion around double bonds. Proceedings of the National Academy of Sciences, 201712784. doi:10.1073/pnas.1712784115

3. Szymański, W., Beierle, J. M., Kistemaker, H. A. V., Velema, W. A., \& Feringa, B. L. (2013). Reversible Photocontrol of Biological Systems by the Incorporation of Molecular Photoswitches. Chemical Reviews, 113(8), 6114-6178. doi:10.1021/cr300179f

4. Schoenlein, R., Peteanu, L., Mathies, R., \& Shank, C. (1991). The first step in vision: femtosecond isomerization of rhodopsin. Science, 254(5030), 412-415.

5. Wald, G. (1968). Molecular Basis of Visual Excitation. Science, 162(3850), 230. doi:10.1126/science.162.3850.230

6. Dartnall, H. (1968). The photosensitivities of visual pigments in the presence of hydroxylamine. Vision research, 8(4), 339-358. 
7. Becker, R. S., \& Freedman, K. (1985). A comprehensive investigation of the mechanism and photophysics of isomerization of a protonated and unprotonated Schiff base of 11-cisretinal. Journal of the American Chemical Society, 107(6), 1477-1485. doi:10.1021/ja00292a005

8. Koyama, Y., Kubo, K., Komori, M., Yasuda, H., \& Mukai, Y. (1991). EFFECT OF PROTONATION ON THE ISOMERIZATION PROPERTIES OF n-BUTYLAMINE SCHIFF BASE OF ISOMERIC RETINAL AS REVEALED BY DIRECT HPLC ANALYSES: SELECTION OF ISOMERIZATION PATHWAYS BY RETINAL PROTEINS. Photochemistry and Photobiology, 54(3), 433-443. doi:10.1111/j.17511097.1991.tb02038.x

9. Kandori, H., Katsuta, Y., Ito, M., \& Sasabe, H. (1995). Femtosecond fluorescence study of the rhodopsin chromophore in solution. Journal of the American Chemical Society, 117(9), 2669-2670. doi:10.1021/ja00114a040

10. Bassolino, G., Sovdat, T., Soares Duarte, A., Lim, J. M., Schnedermann, C., Liebel, M., ... Kukura, P. (2015). Barrierless Photoisomerization of 11- cis Retinal Protonated Schiff Base in Solution. Journal of the American Chemical Society, 137(39), 12434-12437. doi:10.1021/jacs.5b06492

11. Wang, Q., Schoenlein, R., Peteanu, L., Mathies, R., \& Shank, C. (1994). Vibrationally coherent photochemistry in the femtosecond primary event of vision. Science, 266(5184), 422. doi:10.1126/science.7939680

12. Schnedermann, C., Liebel, M., \& Kukura, P. (2015). Mode-Specificity of Vibrationally Coherent Internal Conversion in Rhodopsin during the Primary Visual Event. Journal of the American Chemical Society, 137(8), 2886-2891. doi:10.1021/ja508941k

13. Johnson, P. J. M., Halpin, A., Morizumi, T., Prokhorenko, V. I., Ernst, O. P., \& Miller, R. J. D. (2015). Local vibrational coherences drive the primary photochemistry of vision. Nature Chemistry, 7(12), 980-986. doi:10.1038/nchem.2398

14. Schnedermann, C., Yang, X., Liebel, M., Spillane, K. M., Lugtenburg, J., Fernández, I., ... Mathies, R. A. (2018). Evidence for a vibrational phase-dependent isotope effect on the photochemistry of vision. Nature Chemistry, 10(4), 449-455. doi:10.1038/s41557018-0014-y

15. Weingart, O., Schapiro, I., \& Buss, V. (2007). Photochemistry of Visual Pigment Chromophore Models by Ab Initio Molecular Dynamics. The Journal of Physical Chemistry B, 111(14), 3782-3788. doi:10.1021/jp0683216

16. Schapiro, I., Ryazantsev, M. N., Frutos, L. M., Ferré, N., Lindh, R., \& Olivucci, M. (2011). The Ultrafast Photoisomerizations of Rhodopsin and Bathorhodopsin Are Modulated by Bond Length Alternation and HOOP Driven Electronic Effects. Journal of the American Chemical Society, 133(10), 3354-3364. doi:10.1021/ja1056196

17. Zanirato, V., Pollini, G. P., De Risi, C., Valente, F., Melloni, A., Fusi, S., ... Olivucci, M. (2007). Synthesis of biomimetic light-driven molecular switches via a cyclopropyl ringopening/nitrilium ion ring-closing tandem reaction. Tetrahedron, 63(23), 4975-4982. doi:10.1016/j.tet.2007.03.141

18. Lumento, F., Zanirato, V., Fusi, S., Busi, E., Latterini, L., Elisei, F., ... Olivucci, M. (2007). Quantum Chemical Modeling and Preparation of a Biomimetic Photochemical Switch. Angewandte Chemie International Edition, 46(3), 414-420. doi:10.1002/anie.200602915

19. Sinicropi, A., Martin, E., Ryazantsev, M., Helbing, J., Briand, J., Sharma, D., ... Olivucci, M. (2008). An artificial molecular switch that mimics the visual pigment and completes its photocycle in picoseconds. Proceedings of the National Academy of Sciences, 105(46), 17642. doi:10.1073/pnas.0802376105 
20. Melloni, A., Paccani, R. R., Donati, D., Zanirato, V., Sinicropi, A., Parisi, M. L., ... Frutos, L. M. (2010). Modeling, preparation, and characterization of a dipole moment switch driven by Z/E photoisomerization. Journal of the American Chemical Society, 132(27), 9310.

21. Léonard, J., Schapiro, I., Briand, J., Fusi, S., Paccani, R. R., Olivucci, M., \& Haacke, S. (2012). Mechanistic Origin of the Vibrational Coherence Accompanying the Photoreaction of Biomimetic Molecular Switches. Chemistry - A European Journal, 18(48), 15296-15304. doi:10.1002/chem.201201430

22. Schapiro, I., Fusi, S., Olivucci, M., Andruniów, T., Sasidharanpillai, S., \& Loppnow, G. R. (2014). Initial Excited-State Dynamics of an $N$-Alkylated Indanylidene-Pyrroline (NAIP) Rhodopsin Analog. The Journal of Physical Chemistry B, 118(42), 12243-12250. doi:10.1021/jp508060z

23. Gueye, M., Manathunga, M., Agathangelou, D., Orozco, Y., Paolino, M., Fusi, S., ... Léonard, J. (2018). Engineering the vibrational coherence of vision into a synthetic molecular device. Nature Communications, 9(1), 313. doi:10.1038/s41467-017-02668-w

24. Briand, J., Bräm, O., Réhault, J., Léonard, J., Cannizzo, A., Chergui, M., ... Haacke, S. (2010). Coherent ultrafast torsional motion and isomerization of a biomimetic dipolar photoswitch. Physical Chemistry Chemical Physics, 12(13), 3178. doi:10.1039/b918603d

25. Polli, D., Altoè, P., Weingart, O., Spillane, K. M., Manzoni, C., Brida, D., ... Cerullo, G. (2010). Conical intersection dynamics of the primary photoisomerization event in vision. Nature, 467(7314), 440-443. doi:10.1038/nature09346

26. footnote. (s. d.). Here we take the opportunity to confirm that the QY of Z-MeO-NAIP is $21 \%$ and not $0.20 \%$ as writen by mistake in our previous work ref [23].

27. Filatov, M., \& Olivucci, M. (2014). Designing Conical Intersections for Light-Driven Single Molecule Rotary Motors: From Precessional to Axial Motion. The Journal of Organic Chemistry, 79(8), 3587-3600. doi:10.1021/jo5004289

28. Scholes, G. D., Fleming, G. R., Chen, L. X., Aspuru-Guzik, A., Buchleitner, A., Coker, D. F., ... Zhu, X. (2017). Using coherence to enhance function in chemical and biophysical systems. Nature, 543(7647), 647-656. doi:10.1038/nature21425

29. Blanco-Lomas, M., Samanta, S., Campos, P. J., Woolley, G. A., \& Sampedro, D. (2012). Reversible Photocontrol of Peptide Conformation with a Rhodopsin-like Photoswitch. Journal of the American Chemical Society, 134(16), 6960-6963. doi:10.1021/ja301868p

30. García-Iriepa, C., Gueye, M., Léonard, J., Martínez-López, D., Campos, P. J., Frutos, L. M., ... Marazzi, M. (2016). A biomimetic molecular switch at work: coupling photoisomerization dynamics to peptide structural rearrangement. Phys. Chem. Chem. Phys., 18(9), 6742-6753. doi:10.1039/C5CP07599H

31. Sampedro, D., Migani, A., Pepi, A., Busi, E., Basosi, R., Latterini, L., ... Olivucci, M. (2004). Design and Photochemical Characterization of a Biomimetic Light-Driven Z / E Switcher. Journal of the American Chemical Society, 126(30), 9349-9359. doi:10.1021/ja038859e

32. García-Iriepa, C., Marazzi, M., Zapata, F., Valentini, A., Sampedro, D., \& Frutos, L. M. (2013). Chiral Hydrogen Bond Environment Providing Unidirectional Rotation in Photoactive Molecular Motors. The Journal of Physical Chemistry Letters, 4(9), 1389-1396. doi:10.1021/jz302152v

33. Marchand, G., Eng, J., Schapiro, I., Valentini, A., Frutos, L. M., Pieri, E., ... Gindensperger, E. (2015). Directionality of Double-Bond Photoisomerization Dynamics Induced by a Single Stereogenic Center. The Journal of Physical Chemistry Letters, 6(4), 599-604. doi:10.1021/jz502644h 
34. Schapiro, I., Gueye, M., Paolino, M., Fusi, S., Haacke, S., Marchand, G., ... Olivucci, M. (2019). Synthesis, Spectroscopy and QM/MM Simulations of a Bio-mimetic Ultrafast Light-Driven Molecular Motor. Submitted.

35. Bonačić-Koutecký, V., Köhler, J., \& Michl, J. (1984). Prediction of structural and environmental effects on the S1 $\square$ S0 energy gap and jump probability in double-bond cis - trans photoisomeriz. Chemical physics letters, 104(5), 440-443.

36. Dunkelberger, A. D., Kieda, R. D., Shin, J. Y., Rossi Paccani, R., Fusi, S., Olivucci, M., \& Fleming Crim, F. (2012). Photoisomerization and Relaxation Dynamics of a Structurally Modified Biomimetic Photoswitch. The Journal of Physical Chemistry A, 116(14), 3527-3533. doi:10.1021/jp300153a

37. Briand, J. (2009). Ultrafast isomerization and vibrational coherence of biomiemetic photoswitches: Experimental investigation by femtosecond transient absorption spectroscopy ( $\mathrm{PhD}$ Thesis). Strasbourg.

38. Gueye, M., Nillon, J., Crégut, O., \& Léonard, J. (2016). Broadband UV-Vis vibrational coherence spectrometer based on a hollow fiber compressor. Review of Scientific Instruments, 87(9), 093109. doi:10.1063/1.4962699

39. Paolino, M., Gueye, M., Pieri, E., Manathunga, M., Fusi, S., Cappelli, A., ... Olivucci, M. (2016). Design, Synthesis, and Dynamics of a Green Fluorescent Protein Fluorophore Mimic with an Ultrafast Switching Function. Journal of the American Chemical Society, 138(31), 9807-9825. doi:10.1021/jacs.5b10812

40. Stephens, P. J., Devlin, F. J., Chabalowski, C. F., \& Frisch, M. J. (1994). Ab Initio Calculation of Vibrational Absorption and Circular Dichroism Spectra Using Density Functional Force Fields. The Journal of Physical Chemistry, 98(45), 11623-11627. doi:10.1021/j100096a001

41. Grimme, S. (2006). Semiempirical GGA-type density functional constructed with a longrange dispersion correction. Journal of Computational Chemistry, 27(15), 1787-1799. doi:10.1002/jcc.20495

42. Scalmani, G., \& Frisch, M. J. (2010). Continuous surface charge polarizable continuum models of solvation. I. General formalism. The Journal of Chemical Physics, 132(11), 114110. doi:10.1063/1.3359469

43. Gaussian09. (2009). M. J. Frisch, G. W. Trucks, H. B. Schlegel, G. E. Scuseria, M. A. Robb, J. R. Cheeseman, G. Scalmani, V. Barone, B. Mennucci, G. A. Petersson et al. (Gaussian, Inc., Wallingford CT, 2009).

44. Léonard, J., Briand, J., Fusi, S., Zanirato, V., Olivucci, M., \& Haacke, S. (2013). Isomerdependent vibrational coherence in ultrafast photoisomerization. New Journal of Physics, 15(10), 105022. doi:10.1088/1367-2630/15/10/105022

45. Manathunga, M., Yang, X., Orozco-Gonzalez, Y., \& Olivucci, M. (2017). Impact of Electronic State Mixing on the Photoisomerization Time Scale of the Retinal Chromophore. The Journal of Physical Chemistry Letters, 8(20), 5222-5227. doi:10.1021/acs.jpclett.7b02344

46. Schoenlein, R., Peteanu, L., Wang, Q., Mathies, R., \& Shank, C. (1993). Femtosecond dynamics of cis-trans isomerization in a visual pigment analog: isorhodopsin. The Journal of Physical Chemistry, 97(46), 12087-12092.

47. Sovdat, T., Bassolino, G., Liebel, M., Schnedermann, C., Fletcher, S. P., \& Kukura, P. (2012). Backbone Modification of Retinal Induces Protein-like Excited State Dynamics in Solution. Journal of the American Chemical Society, 134(20), 8318-8320. doi:10.1021/ja3007929

48. Bassolino, G., Sovdat, T., Liebel, M., Schnedermann, C., Odell, B., Claridge, T. D. W., ... Fletcher, S. P. (2014). Synthetic Control of Retinal Photochemistry and Photophysics 
in Solution. Journal of the American Chemical Society, 136(6), 2650-2658. doi: $10.1021 / \mathrm{ja} 4121814$

49. Levine, B. G., \& Martínez, T. J. (2007). Isomerization Through Conical Intersections. Annual Review of Physical Chemistry, 58(1), 613-634. doi:10.1146/annurev.physchem.57.032905.104612 